\title{
NMDA receptor activation stimulates transcription-independent rapid wnt5a protein synthesis via the MAPK signaling pathway
}

\author{
Yichen $\mathrm{Li}^{1 \dagger}$, Bei Li ${ }^{2 \dagger}$, Xianzi Wan ${ }^{1}$, Wei Zhang ${ }^{1}$, Ling Zhong ${ }^{1 *}$ and Shao-Jun Tang ${ }^{2^{*}}$
}

\begin{abstract}
Wht proteins are emerging key regulators of the plasticity and functions of adult brains. However, the mechanisms by which the expression of Wnt proteins is regulated in neurons are unclear. Using cortical primary cultures, we show here that activation of NMDA receptors (NMDARs) induces rapid Wnt5a protein synthesis and secretion. This NMDAR-regulated Wnt5a synthesis does not require transcription and is a result of activity-dependent translation. We also show that NMDAR-regulated Wnt5a translation depends on MAPK signaling but not mTOR signaling. Our findings suggest that the synaptic activity of CNS neurons activates NMDARs, which in turn stimulate translation from stored Wnt5a mRNA via the MAPK signaling pathway.
\end{abstract}

Keywords: activity-regulated protein synthesis, Wnt protein, mTOR signaling, MAPK signaling, NMDA receptors

\section{Background}

Wnts are secreted glycoproteins that regulate cell morphologies and behaviors by stimulating complicate intracellular signaling cascades. Previous work has established that Wnt signaling controls many oncogenic and developmental processes [1,2]. More recent studies have revealed that Wnt signaling is critically involved in key processes of the formation and plasticity of the nervous system, including neurogenesis [3], axon guidance [4], dendritic development [5], synaptic differentiation [6] and plasticity $[7,8]$. Abnormalities of Wnt signaling are implicated in major brain disorders such as Alzheimer's disease [9-11], Parkinson's disease [12,13], schizophrenia $[14,15]$, and drug abuse [16]. Wnt5a is member of the Wnt protein family and plays important roles in outgrowth, guidance and branching of axons $[17,18]$; genesis of dopaminergic neurons [19]; and formation and plasticity of both excitatory and inhibitory synapses [20-22]. Wnt5a administration was reported to improve

\footnotetext{
* Correspondence: Isszhl@mail.sysu.edu.cn; shtang@utmb.edu

+ Contributed equally

${ }^{1}$ School of Pharmaceutical Sciences and Laboratory Animal Center of Sun Yat-Sen University, Guangzhen, P. R. China

${ }^{2}$ Department of Neuroscience and Cell Biology, University of Texas Medical Branch, Galveston, TX77555, USA

Full list of author information is available at the end of the article
}

specific pathological processes of Alzheimer's [11] and Parkinson's diseases in animal models [12].

Wnt proteins bind to receptors to activate the $\mathrm{Wnt} / \beta$ catenin canonical pathway and $\beta$-catenin-independent non-canonical pathways, which include the planar cell polarity (PCP) pathway and the Wnt/calcium $\left(\mathrm{Ca}^{2+}\right)$ pathway [2,23-26]. In the canonical pathway, Wnts (such as Wnt3a) inhibit glycogen synthase kinase $3 \beta$ (GSK-3 $\beta$ ) and consequently stabilize $\beta$-catenin to regulate transcription [1]. Wnt5a is a prototypic Wnt ligand that activates the non-canonical pathways $[27,28]$. The activation of the PCP pathway stimulates Rho GTPases and c-Jun $\mathrm{N}$-terminal kinase (JNK) to regulate cell morphogenesis and movement [29], whereas the activation of the Wnt/ $\mathrm{Ca}^{2+}$ pathway causes $\mathrm{Ca}^{2+}$ to activate protein kinase $\mathrm{C}$ (PKC) and calcium/calmodulin dependent protein kinase II (CaMKII) [30]. In neurons, Wnt secretion is intimately governed by synaptic activity, especially the activation of NMDA receptors (NMDAR) [7].

In contrast to the detailed understanding of the intracellular signaling cascades initiated by Wnts, little is known about the upstream mechanisms that control the synthesis of Wnt proteins. Wayman et al. recently showed that NMDAR activation stimulates CREBmediated Wnt2 transcription [31]. 
We report here a mechanism that couples NMDAR activation to Wnt5a protein synthesis in primary cortical cultures. We observed that NMDAR activation elicited rapid increase and secretion of Wnt5a protein. This NMDAR-regulated Wnt5a protein increase was blocked by translational but not transcriptional inhibitors. In addition, mitogen-activated protein kinase (MAPK) but not mammalian target of rapamycin (mTOR) inhibitors abolished this Wnt5a synthesis. Our findings suggest that a NMDAR/MAPK pathway controls the activity-regulated translation of Wnt5a mRNA in cortical neurons.

\section{Results}

\section{NMDA receptor (NMDAR) activation rapidly increases} Wnt5a in cortical cultures

In an attempt to understand the regulation of Wnt5a expression by synaptic activity, we performed doubleimmunofluorescent staining of Wnt5a and synapsin I (a synaptic marker) to determine the cellular distribution of Wnt5a in mature cortical neurons (12 DIV). The specificity of the anti-Wnt5a antibody was confirmed with a Wnt5a knockout mouse. The results show that Wnt5a is localized in a somato-dendritic pattern (Figure 1A). In dendrites, Wnt5a is detected in regions adjacent to synapsin I signals, indicating a localization of Wnt5a nearby synapses. Next, we sought to determine whether Wnt5a protein expression is regulated by synaptic activity. Western blotting analysis of intracellular proteins indicated that glutamate stimulation ( $10 \mu \mathrm{M}$ for $15 \mathrm{~min})$ stimulation increased Wnt5a in cortical cultures by 4 fold (Figure 1B). Furthermore, NMDA stimulation ( $50 \mu \mathrm{M}$ for $15 \mathrm{~min})$ to activate NMDARs also increased Wnt5a protein by 3.5 fold (Figure 1B). The NMDA-induced Wnt5a increase was completely abolished by DAP5, a specific antagonist of NMDARs (Figure 1C), demonstrating that NMDA indeed elicited Wnt5a protein expression via the activation of NMDARs. These results indicate that NMDAR activation is sufficient to stimulate Wnt5a up-regulation. To characterize the kinetics of NMDAR-dependent Wnt5a protein expression, we determined the time course of NMDA stimulation. As shown in Figure 1D, Wnt5a protein was markedly increased within 5 min after NMDA administration. This observation suggested that NMDAR activation caused rapid Wnt5a synthesis. Strikingly, this increase of intracellular Wnt5a disappeared 30 min after NMDA stimulation (Figure 1D). Because NMDAR activation can evoke Wnt secretion (8), Wnt5a may be secreted to the medium after NMDA stimulation. To test this idea, we performed immunoblotting analysis of Wnt5a in culture media collected at 2, 4, 8, 16, or 32 min after NMDA stimulation. We observed that Wnt5a levels in media increased dramatically after $16 \mathrm{~min}$ (Figure 1E). This data indicates that NMDA activation increases not only the synthesis but also the secretion of Wnt5a. It appears that newly synthesized Wnt5a needs 8-16 min to complete the trafficking process for secretion.

\section{NMDAR-elicited Wnt5a increase requires translation but not transcription}

Given the importance of Wnt5a and NMDAR in the regulation of synaptic plasticity, we were interested in elucidating the mechanism by which NMDAR activation rapidly increases the intracellular Wnt5a concentration in cortical cultures. First, we tested the hypothesis that NMDAR activation caused Wnt5a increase by stimulating mRNA translation. To this end, we used the translation inhibitor, anisomycin $(20 \mu \mathrm{M})$. We observed that pre-treatment of the cultures with anisomycin for 30 min before NMDA application completely abolished the Wnt5a increase elicited by NMDA stimulation ( $50 \mu \mathrm{M} ; 15 \mathrm{~min}$ ) (Figure 2A). This result suggests that NMDAR activation stimulates Wnt5a production via de novo protein synthesis. Because mRNA translation is often coupled with gene transcription, we further tested the hypothesis that NMDARs up-regulate Wnt5a protein production via transcriptional activation. To this end, we used the transcription inhibitor, actinomycin D $(25 \mu \mathrm{M})$. The cultures were pretreated with actinomycin D for 30 min before NMDA application. To our surprise, actinomycin D completely failed to block the Wnt5a increase (Figure 2B). In fact, actinomycin D appeared to increase Wnt5a in this short time window, which might be due to a stimulating effect of actinomycin $\mathrm{D}$ on translation [32]. This observation suggests that NMDARs evoke the rapid Wnt5a protein increase in a transcription-independent process. To verify this notion, we performed quantitative RT-PCR to compare Wnt5a mRNA levels in cultures with or without NMDA stimulation (15 min). No significant differences of Wnt5a mRNA levels were observed in control and treated cultures (Figure $2 \mathrm{C}, \mathrm{D})$. To confirm this observation, we also perform semi-quantitative RT-PCR. As shown in Figure 2E, no obvious difference was detected in the amount of the Wnt5a RT-PCR products from control and NMDA-stimulated cells. Collectively, results from this set of experiments suggest that NMDAR activation evokes rapid translation from pre-existing Wnt5a mRNA in neurons.

mTOR signaling pathway is not required for the NMDARdependent Wnt5a protein synthesis

Previous studies have revealed that mTOR signaling is a major molecular pathway in the control of activity-regulated protein synthesis during synaptic plasticity [33-35]. The mTOR pathway is known to mediate NMDARdependent $\alpha$ CaMKII protein synthesis in hippocampal neurons [35]. And we have found that NMDAR stimulation induced phosphor-P70S6K (p-P70S6K, a downstream target of mTOR signaling) increase, this effect could be diminished by DAP5 (Figure 1C). Therefore, 


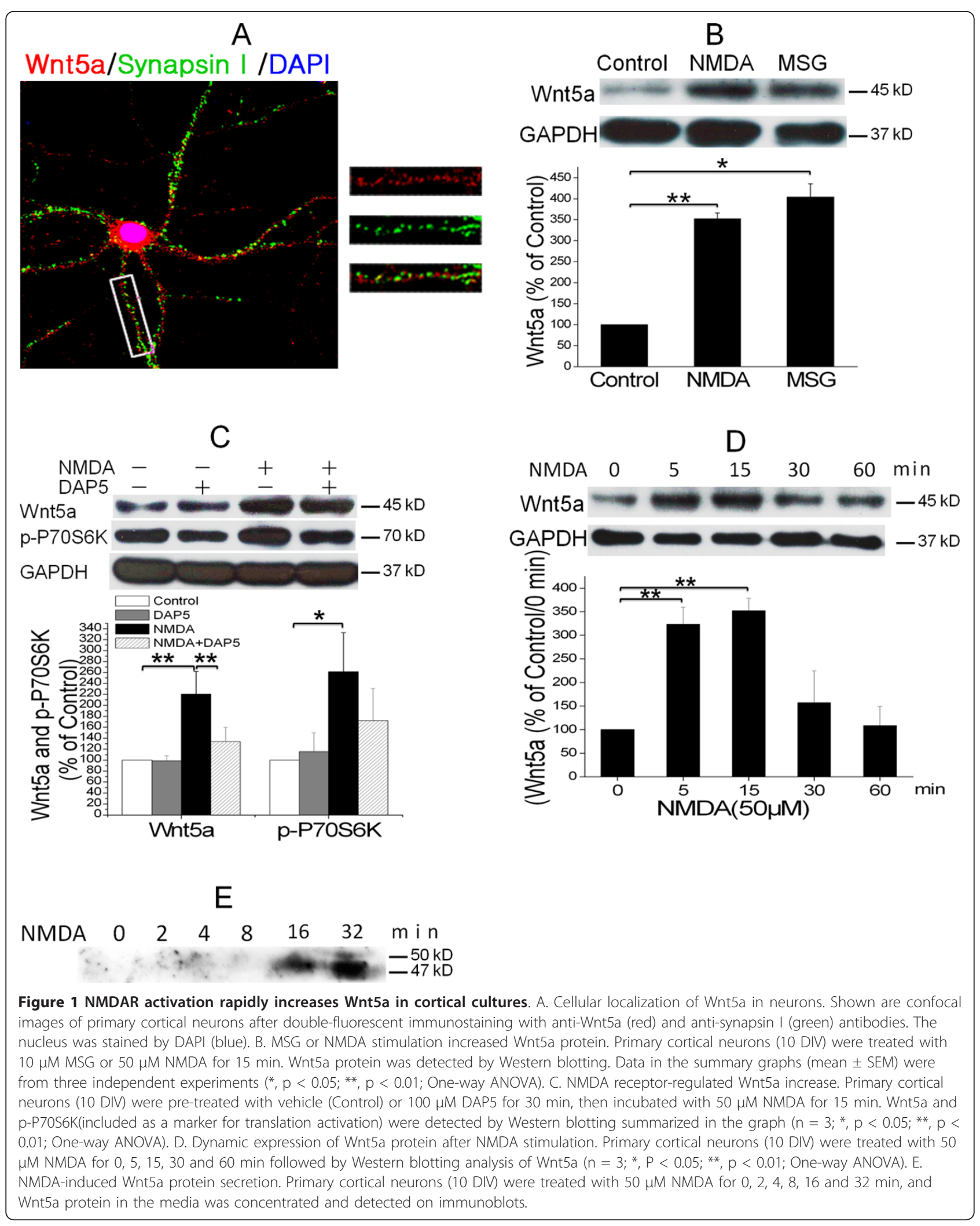




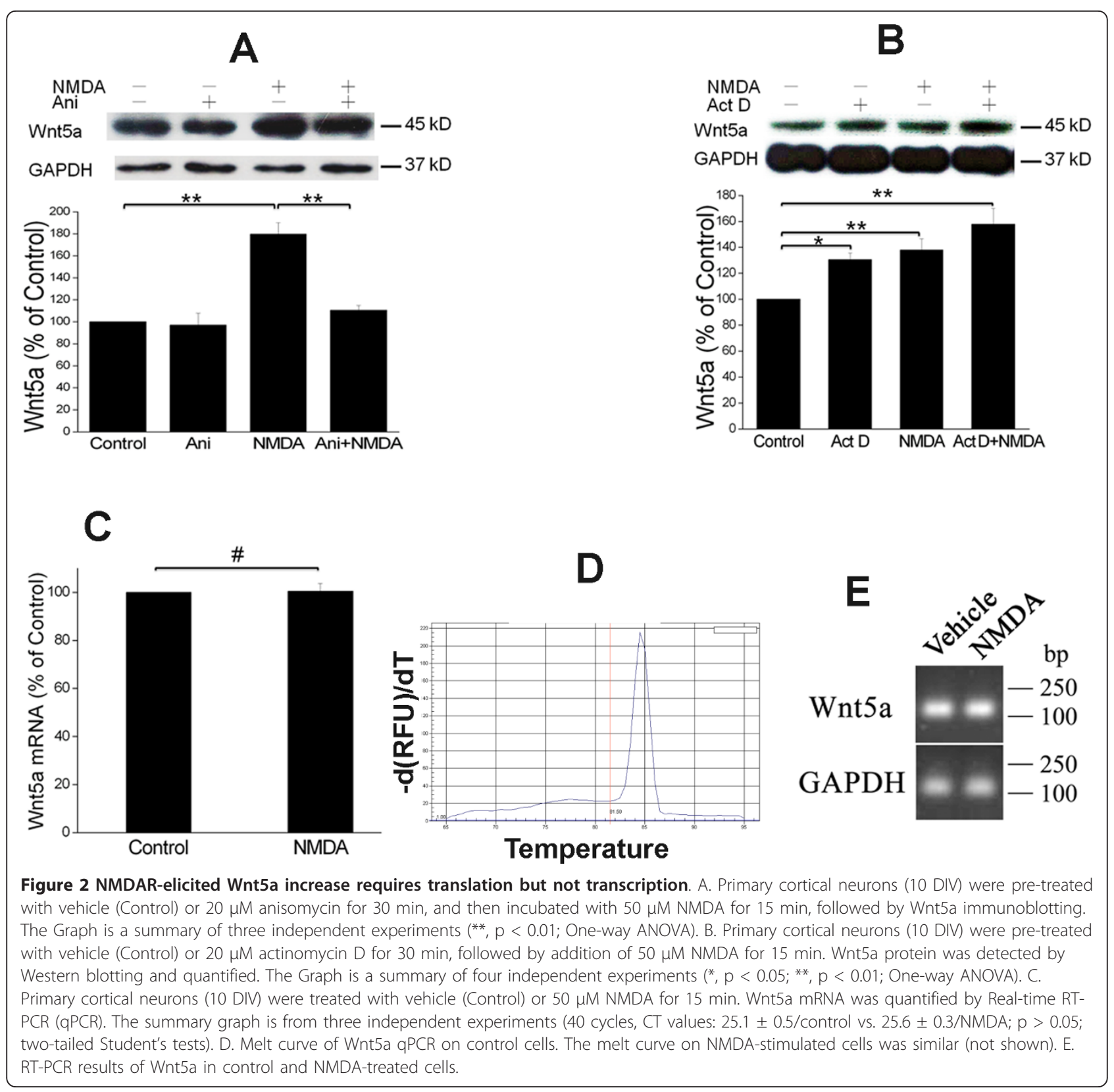

we tested the potential role of mTOR in NMDARinduced Wnt5a translation. Interesting, we found that rapamycin ( $25 \mathrm{nM}, 30 \mathrm{~min}$ of pretreatment), a specific inhibitor of mTOR kinase, did not affect NMDAinduced $(50 \mu \mathrm{M} ; 15 \mathrm{~min}) \mathrm{Wnt} 5 \mathrm{a}$ protein increase (Figure 3). To rule out the possibility of experimental failures, we determined the effect of NMDA and rapamycin on the phosphorylation level of P70S6K. The results showed that NMDA treatment clearly increased p-P70S6K; this increase was abolished by rapamycin (Figure 3), indicating that NMDA activated mTOR signaling and that rapamycin was able to block this activation in our experimental systems. Thus, based on these results, we concluded that the NMDAR-dependent Wnt5a protein synthesis is not mediated by the mTOR signaling pathway.

\section{NMDAR activation stimulates Wnt5a protein synthesis via the MAPK signaling pathway}

Previous studies indicate that MAPK signaling is critical for activity-regulated protein synthesis in neurons [36,37]. We investigated the involvement of MAPK signaling in NMDAR-dependent Wnt5a protein synthesis using pharmacological approaches. We observed that PD98059 (at $20 \mu \mathrm{M}$ for $30 \mathrm{~min}$ pretreatment), a specific MEK inhibitor, blocked the NMDA-evoked Wnt5a increase (Figure 4A). 


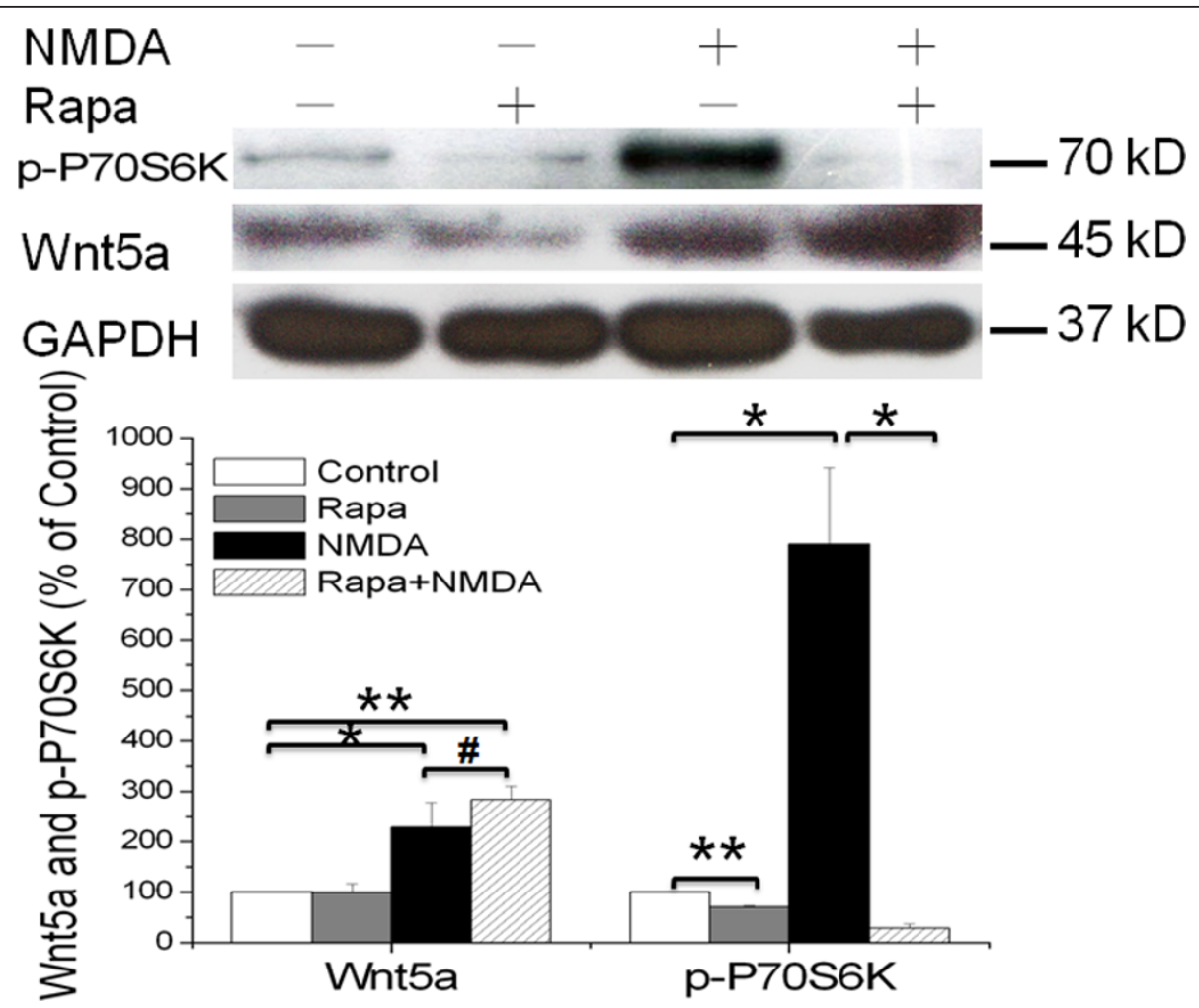

Figure 3 mTOR signaling pathway is not required for the NMDAR-dependent Wnt5a protein synthesis. Primary cortical neurons (10 DIV) were pre-treated with vehicle (Control) or $25 \mathrm{nM}$ Rapamycin for 30 min, followed by addition of $50 \mu \mathrm{M}$ NMDA for 15 min. Western blotting analysis of Wnt5a and phosphor-P70S6K proteins were performed. Graphs (mean \pm SEM) are from four independent experiments $\left({ }^{*}, p<0.05 ;{ }^{* *}\right.$, $p<0.01 ; \#, p>0.05 ;$ One-way ANOVA).

To confirm this observation, we employed another MEK inhibitor, U0126, and we found that U0126 $(20 \mu \mathrm{M}, 30$ min pretreatment) also diminished the NMDA-induced Wnt5a protein increase (Figure 4B). These findings strongly suggest that the MAPK signaling pathway is essential for NMDAR to activate Wnt5a translation.

\section{Conclusion and Discussion}

In this study, we found that NMDAR activation rapidly increases the synthesis of Wnt5a protein. We further elucidate that the NMDAR-regulated rapid Wnt5a synthesis depends on translation but not transcription and that NMDAR-induced translation from the preexisting Wnt5a
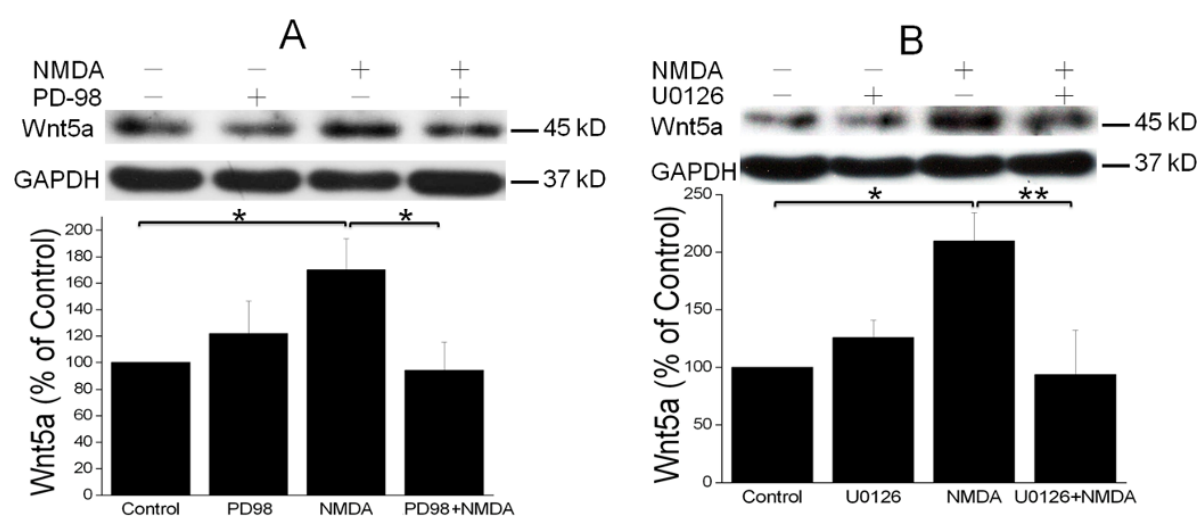

Figure 4 NMDAR activation stimulates Wnt5a protein synthesis via the MAPK signaling pathway. A. Primary cortical neurons (10 DIV) were pre-treated with vehicle (Control) or $20 \mu \mathrm{M}$ PD98059 (PD98) for 30 min, and then stimulated with $50 \mu \mathrm{M}$ NMDA for 15 min. Wnt5a protein was measured by Western blotting and quantified data were presented in graphs (mean $\pm \mathrm{SEM} ; \mathrm{n}=3$, ${ }^{*} \mathrm{p}<0.05$; One-way ANOVA). B. Primary cortical neurons (10 DIV) were pre-treated with vehicle (Control) or $20 \mu \mathrm{M}$ U0126 for $30 \mathrm{~min}$, followed by $50 \mu \mathrm{M}$ NMDA for 15 min. Graphs (mean \pm SEM) are from three independent experiments $\left({ }^{*}, p<0.05 ;{ }^{*}, p<0.01\right.$; One-way ANOVA). 
mRNA is activated by MAPK signaling but not the mTOR signaling pathway.

Inestrosa and co-workers showed that Wnt5a modulates the plasticity of both glutamatergic and GABAergic synapses on hippocampal neurons [20,21]. However, the mechanism of Wnt5a regulation during the induction and expression of synaptic plasticity was not known. Our findings reveal that synaptic activity, via NMDAR activation, stimulates the synthesis of Wnt5a protein. Because Wnt5a is in dendritic regions-near the presynaptic terminals in mature neurons (Figure 1A)-the rapid synthesis and secretion of Wnt5a following NMDAR activation probably provide an endogenous source of Wnt5a to alter the molecular organization and function of synapses. Indeed, Chen et al. reported that NMDAR-dependent secretion of Wnt3a regulates synaptic plasticity in hippocampal slices [7]. These findings collectively support the view that activity-regulated synthesis and secretion of Wnts are basic molecular processes underlying the expression of synaptic plasticity [8].

The increase in NMDAR-regulated Wnt5a protein is a result of de novo translation that does not require mRNA transcription (Figure 2A, C). These findings indicate that there is dormant Wnt5a mRNA stored in neurons, and this mRNA is positioned for translational initiation following NMDAR activation. This provides a mechanism for neurons to quickly generate new Wnt5a, which is probably needed for synaptic processes that are critical in the early stage of synaptic plasticity soon after synaptic activation, including the re-organization of synaptic proteins [20,21]. On the other hand, Wayman et al. showed that in differentiating hippocampal neurons NMDAR activation stimulates Wnt2 transcription, which regulates dendritic arborization [31]. Together, these findings indicate that NMDARs may evoke the expression of different Wnt proteins by stimulating either transcription or translation in different cellular contexts.

The mTOR signaling pathway is a key mechanism by which synaptic activity stimulates protein synthesis in neurons $[38,39]$. However, our results indicate that this pathway is not involved in the activation of NMDARregulated Wnt5a mRNA translation (Figure 4A, B). Instead, the NMDAR-elicited Wnt5a protein synthesis requires the activation of the MAPK signaling pathway. Tsokas et al. reported that MAPK signaling can stimulate activity-regulated synthesis of translational proteins by controlling the mTOR signaling pathway [40]. Because mTOR is not required for Wnt5a synthesis (Figure 3), we conclude that MAPK signaling leads to translational activation via an mTOR signaling-independent pathway.

Based on the results presented here, we propose the following model: In resting neurons, Wnt5a mRNAs are stored in a translationally inactive form. When neurons are stimulated, synaptic activity induces $\mathrm{Ca}^{2+}$ influx through NMDARs to activate MAPKs to elicit de novo Wnt5a mRNA translation (Figure 5).

\section{Materials and methods Compounds}

NMDA [N-methyl-D-aspartic acid, product number (M3262)], DAP5 [D(-)-2-Amino-5-phosphonovaleric acid, A8054], Poly-D-lysine (P7280), U0126 (U120), Trypsin 10x solution (T4674), MSG (L-glutamic acid monosodium salt hydrate, G5889), Rapamycin (R8781), PD98059 (P215), Actinomycin D (A4262); Anisomycin (A9789) were purchased from Sigma; DAPI (s36939) from invitrogen; HBSS (Hank's balanced salt solution 10×, 14185), D-MEM/F-12 (Dulbecco's modified eagle medium: nutrient mixture F-12, 12400-024), L-Glutamine 100× (25030), B27 50×, NBM (Neurobasal medium, 21103) from Gibco; FBS (Fetal bovine serum, A15-101) from PAA; and DMSO (0231) from Amresco.

NMDA was dissolved in NBM 5 min before treatment. DAP5, U0126, Rapamycin, PD98059, Anisomycin were prepared as $1000 \times$ concentrated stocks in DMSO. All other compounds were prepared as $1000 \times$ concentrated stocks in ultrapure water.

\section{Antibodies}

Anti-Wnt5a antibody was purchased from R\&D Systems (AF654); anti-p-P70S6K (Thr389) antibody from Cell Signaling Technology (\#9206); anti-GAPDH antibody from Santa Cruz (SC-32233); anti-Synapsin I from Millipore (AB1543P); and FITC-conjugated donkey anti-rabbit secondary antibody (711-097-020) and Rhodamineconjugated rabbit anti-goat secondary antibody (305297-003) from Jackson.

\section{Primary cortical culture}

Cortical cultures were prepared as described (32). Briefly, cortices were dissected from C57BL/6J mouse embryos (E18) in HBSS, stripped from blood vessels, and cut into small pieces. They were then digested in $1 \times$ trypsin for 8 min at $37^{\circ}$ in $5 \mathrm{ml}$ tubes and dissociated into single cells by gentle aspirations with a fire-polished glass pipette. After sitting on the bench for $2 \mathrm{~min}$, cells in the supernatant were transferred into fresh tubes and centrifuged for $5 \mathrm{~min}$ (1000 rpm). Cell pellets were suspended in DMEM. Cells were plated on 12-well plates (JETBIOFIL) with poly-Dlysine $(20 \mu \mathrm{g} / \mathrm{ml})$ at a density of $5 \times 105$ cells/well and incubated at $37^{\circ}$ in a humidified atmosphere of $95 \%$ air and $5 \% \mathrm{CO} 2$. One hour later, the culture media were replaced with NBM supplemented with $2 \%$ B27, 5 mM glutamine, $1 \%$ streptomycin and penicillin. The media were changed every three days. Cultures were used for stimulation at day 10 in vitro. 


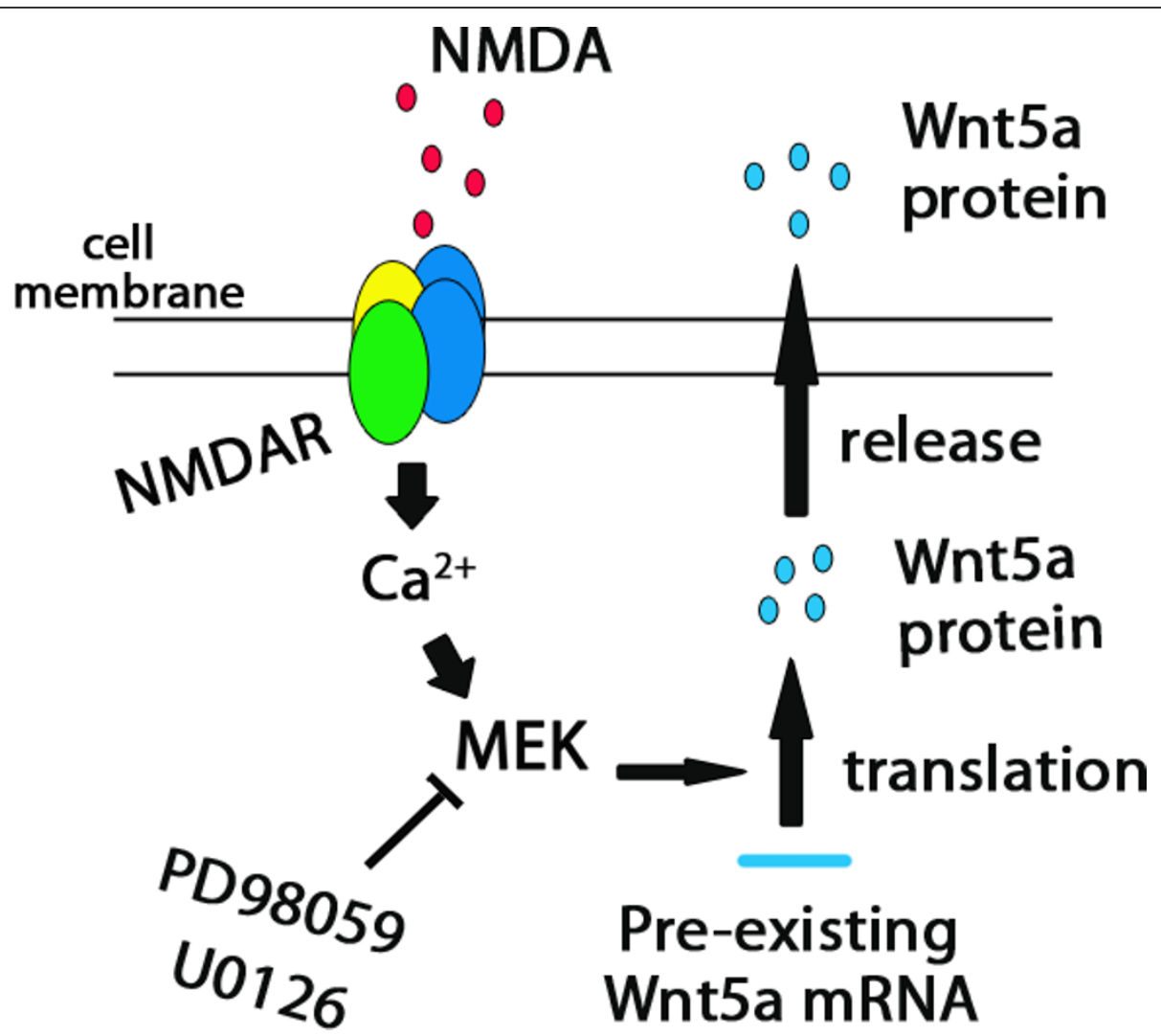

Figure 5 A working model for NMDAR-regulated Wnt5a protein synthesis. Stimulation of NMDAR activates MAPK signaling to elicit de novo translation from pre-existing Wnt5a mRNAs.

Real-time fluorescence quantitative PCR

Cultures (10 DIV; $2 \times 106$ cells/well in 6-well plates) were switched to fresh media for $1 \mathrm{~h}$ and then stimulated with NMDA $(50 \mu \mathrm{M})$ for $15 \mathrm{~min}$ at $37^{\circ}$. Total RNA was purified from the cultures with TRIZOL (invitrogen) according to the manufacturer's instructions. The RNA purity was determined by the OD260/OD280 ratio, and the concentration was calculated based on OD260. The RNA $(1.5 \mu \mathrm{g})$ was used for reverse transcription, followed by quantitative realtime PCR using PrimeScriptTM RT reagent kit (TaKaRa). PCRs (25 $\mu$ l reactions) contained $12.5 \mu \mathrm{l} 2 \times$ SYBR Premix Ex TaqTM, $0.5 \mu \mathrm{l}$ PCR Forward Primer $(10 \mu \mathrm{M}), 0.5 \mu \mathrm{l}$ PCR Reverse Primer $(10 \mu \mathrm{M}), 9.5 \mu \mathrm{l} \mathrm{dH} 2 \mathrm{O}$ and $2 \mu \mathrm{l}$ cDNA. The following primers were employed: Wnt5a Reverse primer: 5'-AGCCAGCACGTCTTGAGGCTA-3'; Wnt5a Forward primer: 5'-AA TCCACGCTAAGGGTTCCTATGAG -3'; $\beta$-actin Reverse: 5'-GCAATGCCTGGGTACATGGT GG-3'; and $\beta$-actin Forward: 5'-ACGCGTCGACCTCCT TGCAGTCCATTTT-3'. PCR was run for one cycle at $95^{\circ}$ for $10 \mathrm{~s}$, and 40 cycles at $95^{\circ}$ for $5 \mathrm{~s} ; 60^{\circ}$ for $20 \mathrm{~s}$.
Immunofluorescent staining

Primary cortical neurons that had been grown on glass coverslips were briefly washed twice with cold PBS, and then fixed in $4 \%$ paraformaldehyde for $30 \mathrm{~min}$ at room temperature (RT). Neurons after fixation were washed with cold PBS $(3 \times 5 \mathrm{~min})$, permeabilized with $0.1 \%$ Triton X-100 for $10 \mathrm{~min}$, rinsed three times, and blocked with $1 \%$ BSA in PBS for $1 \mathrm{~h}$ (RT). Next, neurons were incubated with primary antibodies (double-stained with anti-Wnt5a antibody at 1:50 and anti-synapsin I antibody at $1: 200)$ in $1 \%$ BSA/PBS in a humidified chamber overnight at $4^{\circ}$, rinsed three times in PBS $(3 \times 5 \mathrm{~min})$. This was followed by incubation with secondary antibodies [Rhodamine-conjugated anti rabbit IgG (recognizing anti-Wnt5a antibody) and FITC-conjugated anti goat IgG (recognizing anti-synapsin I antibody)] in 1\% BSA/ PBS in a light-proof container ( $1 \mathrm{~h}$ at $\mathrm{RT})$. Then, cells were washed $(3 \times 5 \mathrm{~min}$ in PBS), stained with $0.1 \mu \mathrm{g} / \mathrm{ml}$ Hoechst for $1 \mathrm{~min}$, and rinsed with PBS before being mounted. 


\section{Western blotting}

To detect intracellular proteins, cortical neurons in 12well plates $(5 \times 105$ cells/well; 10 DIV) were rinsed with PBS and lysed immediately in $100 \mu \mathrm{l}$ of $2 \times$ SDS-PAGE sample buffer (1×: $62.5 \mathrm{mM}$ Tris, pH 6.8, 2\% SDS, 5\% 2 -mercaptoethanol, $10 \%$ glycerol, and $0.0025 \%$ bromophenol blue as described previously [32]). These were then boiled for $10 \mathrm{~min}$. After electrophoresis on 10\% SDS-PAGE gels, proteins were transferred to $0.2 \mu \mathrm{m}$ Immobilon polyvinylidene difluoride (PVDF) membranes (Millipore) and blotted with primary and HRP-conjugated secondary antibodies. The signals were detected using the ECL system (Pierce). To detect secreted Wnt5a, media of cortical neurons in 12-well plates $(1 \times$ 106 cells/well; 10 DIV) were replaced with $300 \mu \mathrm{l} \mathrm{NBM}$ before NMDA stimulation. All NBM was collected after the stimulation and heat-evaporated to a final volume suitable for one loading on an SDS-PAGE gel.

\section{Quantification and statistics}

Immunoblots were scanned with an Epson scanner, and the optical density $(\mathrm{OD})$ of protein bands were quantified with Quantity One software (Bio-Rad). The statistical tests were performed by one-way ANOVA or by two-tailed Student's tests, using SPSS 16.0. Graphs of quantified data (Mean data + SEM) were prepared using Origin.

\section{Acknowledgements}

This work was supported by the UTMB start-up funds and the Whitehall Foundation to S.-J. T., the National Natural Science Foundation of China (No. 30873457 to L. Z. and No. 30873457 to W. Z.) and the Scientific Technology Project of Guangdong Province of China (No.2008A060202010 and No. 2010 B050700019 to L. Z. and 2008A060202010 and 2010B050700019 to W. Z.).

\section{Author details}

${ }^{1}$ School of Pharmaceutical Sciences and Laboratory Animal Center of Sun Yat-Sen University, Guangzhen, P. R. China. ${ }^{2}$ Department of Neuroscience and Cell Biology, University of Texas Medical Branch, Galveston, TX77555, USA

\section{Authors' contributions}

$Y L$ and $B L$ performed experiments, prepared the figures and drafted the manuscript. XW participated in a part of the study. XW provided critical reagents. $L Z$ coordinated the performance of the study and contributed to experimental design and data analysis. SJT conceived of the study and participated in its design and manuscript writing. All authors read and approved the final manuscript.

\section{Competing interests}

The authors declare that they have no competing interests.

Received: 6 October 2011 Accepted: 4 January 2012

Published: 4 January 2012

\section{References}

1. Logan CY, Nusse R: The Wnt signaling pathway in development and disease. Annu Rev Cell Dev Biol 2004, 20:781-810.

2. Ciani $L$, Salinas PC: WNTs in the vertebrate nervous system: from patterning to neuronal connectivity. Nat Rev Neurosci 2005, 6(5):351-62.
3. Lie $D C$, et al: Wnt signalling regulates adult hippocampal neurogenesis. Nature 2005, 437(7063):1370-5.

4. Zou Y: Wnt signaling in axon guidance. Trends Neurosci 2004, 27(9):528-32

5. Rosso SB, et al: Wnt signaling through Dishevelled, Rac and JNK regulates dendritic development. Nat Neurosci 2005, 8(1):34-42.

6. Packard $M$, et al: The Drosophila Wnt, wingless, provides an essential signal for pre- and postsynaptic differentiation. Cell 2002, 111(3):319-30

7. Yang $H$, et al: Optimized and efficient preparation of astrocyte cultures from rat spinal cord. Cytotechnology 2006, 52:87-97.

8. Tang SJ: The synaptic Wnt signaling hypothesis. Synapse 2007, 61(10):866-8.

9. De Ferrari GV, et al: Activation of Wnt signaling rescues neurodegeneration and behavioral impairments induced by betaamyloid fibrils. Mol Psychiatry 2003, 8(2):195-208.

10. Fuentealba RA, et al: Signal transduction during amyloid-beta-peptide neurotoxicity: role in Alzheimer disease. Brain Res Brain Res Rev 2004 47(1-3):275-89

11. Cerpa W, et al: Wnt-5a occludes Abeta oligomer-induced depression of glutamatergic transmission in hippocampal neurons. Mol Neurodegener 2010, 5:3.

12. Parish $\mathrm{CL}$, et al: Wnt5a-treated midbrain neural stem cells improve dopamine cell replacement therapy in parkinsonian mice. J Clin Invest 2008, 118(1):149-60.

13. Rawal N, et al: Parkin protects dopaminergic neurons from excessive Wnt/beta-catenin signaling. Biochem Biophys Res Commun 2009, 388(3):473-8

14. Cotter $D$, et al: Abnormalities of Wnt signalling in schizophreniaevidence for neurodevelopmental abnormality. Neuroreport 1998, 9(7):1379-83.

15. Moon RT, et al: WNT and beta-catenin signalling: diseases and therapies. Nat Rev Genet 2004, 5(9):691-701.

16. Novikova SI, et al: Neuropathology of the cerebral cortex observed in a range of animal models of prenatal cocaine exposure may reflect alterations in genes involved in the Wnt and cadherin systems. Synapse 2005, 56(2):105-16

17. Bodmer D, et al: Wnt5a mediates nerve growth factor-dependent axonal branching and growth in developing sympathetic neurons. J Neurosci 2009, 29(23):7569-81.

18. Li L, Hutchins Bl, Kalil K: Wnt5a induces simultaneous cortical axon outgrowth and repulsive axon guidance through distinct signaling mechanisms. J Neurosci 2009, 29(18):5873-83.

19. Bryja $V$, et al: Wnt-5a induces Dishevelled phosphorylation and dopaminergic differentiation via a CK1-dependent mechanism. J Cell Sci 2007, 120(Pt 4):586-95

20. Farias GG, et al: Wnt-5a/JNK signaling promotes the clustering of PSD-95 in hippocampal neurons. J Biol Chem 2009, 284(23):15857-66.

21. Cuitino $L$, et al: Wnt-5a modulates recycling of functional GABAA receptors on hippocampal neurons. J Neurosci 2010, 30(25):8411-20.

22. Varela-Nallar $\mathrm{L}$, et al: Wingless-type family member $5 \mathrm{~A}$ (Wnt-5a) stimulates synaptic differentiation and function of glutamatergic synapses. Proc Natl Acad Sci USA 2010, 107(49):21164-9.

23. Strutt D: Frizzled signalling and cell polarisation in Drosophila and vertebrates. Development 2003, 130(19):4501-13.

24. Veeman MT, Axelrod JD, Moon RT: A second canon. Functions and mechanisms of beta-catenin-independent Wnt signaling. Dev Cell 2003, 5(3):367-77.

25. Kuhl M: The WNT/calcium pathway: biochemical mediators, tools and future requirements. Front Biosci 2004, 9:967-74

26. Inestrosa NC, Arenas E: Emerging roles of Wnts in the adult nervous system. Nat Rev Neurosci 2010, 11(2):77-86.

27. Du SJ, et al: Identification of distinct classes and functional domains of Wnts through expression of wild-type and chimeric proteins in Xenopus embryos. Mol Cell Biol 1995, 15(5):2625-34.

28. Liu G, Bafico A, Aaronson SA: The mechanism of endogenous receptor activation functionally distinguishes prototype canonical and noncanonical Wnts. Mol Cell Biol 2005, 25(9):3475-82.

29. Katoh M: WNT/PCP signaling pathway and human cancer (review). Oncol Rep 2005, 14(6):1583-8.

30. Sheldahl LC, et al: Dishevelled activates Ca2+ flux, PKC, and CamKII in vertebrate embryos. J Cell Biol 2003, 161(4):769-77. 
31. Wayman GA, et al: Activity-dependent dendritic arborization mediated by CaM-kinase I activation and enhanced CREB-dependent transcription of Wnt-2. Neuron 2006, 50(6):897-909.

32. Loreni $F$, Thomas $G$, Amaldi F: Transcription inhibitors stimulate translation of $5^{\prime}$ TOP mRNAs through activation of S6 kinase and the mTOR/FRAP signalling pathway. Eur J Biochem 2000, 267(22):6594-601.

33. Tang SJ, et al: A rapamycin-sensitive signaling pathway contributes to long-term synaptic plasticity in the hippocampus. Proc Natl Acad Sci USA 2002, 99(1):467-72

34. Asnaghi $L$, et al: mTOR: a protein kinase switching between life and death. Pharmacol Res 2004, 50(6):545-9.

35. Gong R, et al: Roles of glutamate receptors and the mammalian target of rapamycin (mTOR) signaling pathway in activity-dependent dendritic protein synthesis in hippocampal neurons. J Biol Chem 2006, 281(27):18802-15.

36. Kelleher RJ, et al: Translational control by MAPK signaling in long-term synaptic plasticity and memory. Cell 2004, 116(3):467-79.

37. Gong R, Tang SJ: Mitogen-activated protein kinase signaling is essential for activity-dependent dendritic protein synthesis. Neuroreport 2006, 17(15):1575-8.

38. Sutton MA, Schuman EM: Local translational control in dendrites and its role in long-term synaptic plasticity. J Neurobiol 2005, 64(1):116-31.

39. Costa-Mattioli M, et al: Translational control of long-lasting synaptic plasticity and memory. Neuron 2009, 61(1):10-26.

40. Tsokas $P$, et al: Mitogen-activated protein kinase upregulates the dendritic translation machinery in long-term potentiation by controlling the mammalian target of rapamycin pathway. J Neurosci 2007, 27(22):5885-94.

doi:10.1186/1756-6606-5-1

Cite this article as: $L i$ et al: NMDA receptor activation stimulates transcription-independent rapid wnt5a protein synthesis via the MAPK signaling pathway. Molecular Brain 2012 5:1.

\section{Submit your next manuscript to BioMed Central and take full advantage of:}

- Convenient online submission

- Thorough peer review

- No space constraints or color figure charges

- Immediate publication on acceptance

- Inclusion in PubMed, CAS, Scopus and Google Scholar

- Research which is freely available for redistribution

Submit your manuscript at www.biomedcentral.com/submit
Ciomed Central 